\title{
New Concepts of Metallic Bonding Based on Valence-Bond Ideas
}

\author{
Mark H. McAdon and William A. Goddard, III \\ Arthur Amos Noyes Laboratory of Chemical Physics, California Institute of Technology, Pasadena, California 91125 \\ (Received 23 May 1985)
}

\begin{abstract}
From generalized-valence-bond calculations on numerous $\mathrm{Li}$-atom clusters $\left(\mathrm{Li}_{n}\right.$ and $\mathrm{Li}_{n}{ }^{+}$, $n \leqslant 13$, we conclude that the optimum bonding involves singly occupied orbitals localized interstitially (in tetrahedra). Rules based on the calculations are used to predict low-energy isomers (leading for $\mathrm{Li}_{13}{ }^{+}$to low-symmetry structures that are significantly more stable than the icosahedron but retain local fivefold-symmetry axes) and are applied to infinite metallic systems.
\end{abstract}

PACS numbers: $36.40 .+\mathrm{d}, 31.10 .+\mathrm{z}, 31.20 . \mathrm{Tz}, 71.10 .+\mathrm{x}$

The valence-bond principles of structural chemistry,$^{1}$ so useful in rationalizing the geometries and bonding for nonmetallic molecules and solids, ${ }^{2-5}$ have not been similarly useful for understanding metallic systems. In order to lay the foundation for the development of analogous simple principles for metallic systems, we have applied $a b$ initio generalizedvalence-bond (GVB) approaches to examining the bonding in various one-, two-, and three-dimensional clusters of $\mathrm{Li}$ atoms. The qualitative principles arising from these studies are presented here (further details will appear elsewhere). ${ }^{6}$ These principles should be useful in rationalizing and predicting the local geometric and electronic structures for clusters and amorphous metals, ${ }^{6}$ and for defects and interfaces of crystalline metallic systems.

For one-dimensional systems we find one singly occupied orbital localized at each bond midpoint, forming one-electron bonds. This is shown for the $\mathrm{Li}_{10}$ ring cluster in Fig. 1. These bond-localized orbitals are very similar for other lithium rings and for $\mathrm{Li}_{2}{ }^{+}$, linear $\mathrm{Li}_{3}{ }^{+}, \mathrm{Cu}_{2}{ }^{+}$, and linear $\mathrm{Cu}_{3}{ }^{+} .6$ The GVB wave func-

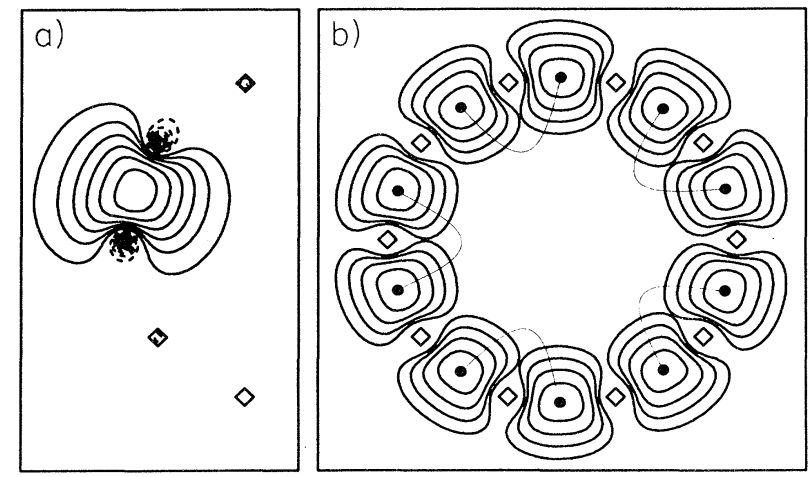

FIG. 1. The GVB orbitals for one of the two primary resonance structures for the $\mathrm{Li}_{10}$ ring cluster. Squares mark the atomic positions and dots joined by thin lines represent the electrons and spin coupling. Solid and dashed contours show positive and negative orbital amplitudes, respectively. Contour spacings are at 0.015 a.u. (a) One singly occupied GVB orbital. (b) Contours $\geqslant 0.045$ a.u. for all ten GVB orbitals. tion of the $\mathrm{Li}_{10}$ ring involves full optimization of the spin coupling (42 possible resonance structures) but is dominated by two primary resonance structures having adjacent (singly occupied) bond orbitals spin paired. This model for the bonding predicts correctly the distortions that occur when the ring structure is allowed to relax. The optimum geometric distortion is the one that increases the overlap of adjacent spin-paired orbitals while decreasing the overlap of adjacent orbitals that are not spin paired. In square $\mathrm{H}_{4}$, the orbitals are atom centered, leading to an unstable rectangular distortion (no chemical bonding between the two $\mathrm{H}_{2}$ fragments). For square $\mathrm{Li}_{4}$, the orbitals are bond centered, leading to a rhombic distortion [Fig. 2(a)] that is unstable as a result of one-electron bonding. Such distortions lower the energy of one resonance structure but raise the energy of the other resonance structure, reducing the resonance energy substantially. Nevertheless, square $\mathrm{Li}_{4}\left(\theta=90^{\circ}\right)$ distorts to a rhombus $s^{6,7}$ $\left(\theta_{\text {opt }}=57.3^{\circ}\right)$. Similarly, hexagonal $\operatorname{Li}_{6}\left(\theta=120^{\circ}\right)$ distorts to a trigonal hexagon ${ }^{6,8}\left(\theta_{\text {opt }}=69.3^{\circ}\right)$, as indicated in Fig. 2(b). Thus, optimum planar structures for $\mathrm{Li}_{4}$ and $\mathrm{Li}_{6}$ both resemble planar close-packed clusters.

Similar studies of larger two-dimensional closepacked arrays of $\mathrm{Li}$ atoms lead to the general result that interior regions have singly occupied orbitals localized at the centers of equilateral triangles while surface (edge) regions have singly occupied orbitals localized at

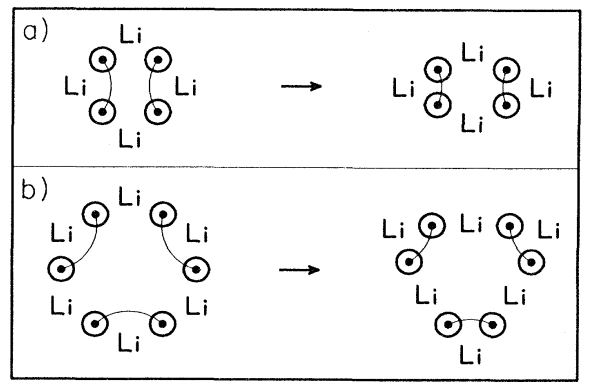

FIG. 2. Geometric distortions for the (a) $\mathrm{Li}_{4}$ and (b) $\mathrm{Li}_{6}$ ring clusters. Circles with dots represent the singly occupied bond orbitals. Lines connecting the dots show the dominant spin pairing. 
bond midpoints. This is illustrated for planar $\mathrm{Li}_{10}$ in Fig. 3.

Use of simple pair-additive potentials would suggest that tetrahedral $\mathrm{Li}_{4}$ is favored over the rhombus since it has one extra "bond." However, the rhombus is the stable geometry as predicted by the following GVB argument. The above results suggest that the four orbitals of tetrahedral $\mathrm{Li}_{4}$ would be localized on the four faces and spin paired. The problem with this is that the orbitals in different pairs would be quite close $(0.41 R$, where $R$ is the side length), leading to large repulsive interactions. Instead, the optimum orbitals are localized on four of the six edges and spin paired so that nonpaired orbitals are at least $0.5 R$ apart. However, the distortion to a planar (rhombic) structure increases the distance between nonpaired orbitals to $0.89 R$, while retaining the favorable spin pairing. Thus the bonding principles predict and the calculations confirm that the stable geometry of $\mathrm{Li}_{4}$ is the rhombus.

We next discuss the lowest-energy spin singlet for $\mathrm{Li}_{13}{ }^{+}$clusters having a central (bulk) atom and twelve surface atoms: (i) $I_{h}$, the icosahedron; (ii) fcc, the truncated octahedron corresponding to the nearest neighbors of a face-centered-cubic lattice; and (iii) hcp, the hexagonal array corresponding to the nearest neighbors of a hexagonal close-packed lattice.

For $I_{h}$, the ground state involves orbitals localized at the centers of twelve of the twenty triangular faces and spin paired in rhombi ( 0.63 overlap) just as for the bulk atoms in Fig. 3. The four triangular faces adjacent to a spin-paired rhombus are empty (to avoid repulsive interactions between unpaired orbitals), as indicated in Fig. 4.

The fcc and hcp clusters have total energies $0.26 \mathrm{eV}$ and $0.56 \mathrm{eV}$ (per cluster) higher than $I_{h}$ (i.e., 20 $\mathrm{meV} /$ atom and $44 \mathrm{meV} /$ atom), respectively. Both have only eight tetrahedra, and some of the tetrahedra have two spin-paired orbitals (on opposite faces). For

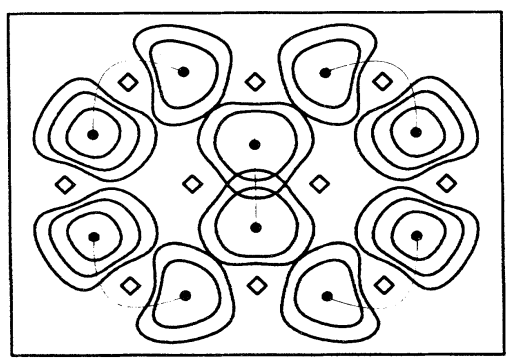

FIG. 3. All ten GVB orbitals for the principal resonance structure of planar close-packed $\mathrm{Li}_{10}$. Squares mark the atoms, and dots joined by thin lines represent the paired electrons. The contours represent 0.015 -a.u. increments of orbital amplitude, starting at 0.060 a.u. Each orbital is occupied by only one electron. fcc, each tetrahedron shares edges (and no faces) with three others and the electrons are distributed so that each edge-sharing pair of tetrahedra contains three electrons. For hcp, there are three pairs of face-shared tetrahedra. This accommodates three pairs of spinpaired orbitals. The top and bottom tetrahedra are isolated and each contains one electron pair. This leaves two electrons that end up along two of the three equatorial edges that do not border a tetrahedron.

From these various results emerge the following rules for three-dimensional metallic structures: $(\alpha)$ Orbitals (each with one electron) are localized in different tetrahedral hollows where possible. ( $\beta$ ) If necessary, two orbitals may be placed in one tetrahedron (localized on opposite faces or edges) but they must be spin paired. $(\gamma)$ No more than three electrons may be distributed between a pair of edgeshared tetrahedra. ( $\delta)$ No more than two electrons may be distributed between a pair of face-shared tetrahedra, and these must be spin paired (singlet). ( $\epsilon$ ) Additional electrons must be in surface orbitals at edge or face sites that do not share edges with occupied tetrahedra. ( $\zeta$ ) It is unfavorable to occupy more than four tetrahedra (or pairs of tetrahedra) sharing one central atom.

We expect that these rules may apply to the valence $s p$ electrons of a variety of structures for elemental metals and alloys, including fcc, hcp, $\gamma$-brass, ${ }^{9,10}$ the Laves phases, ${ }^{10}$ and Frank-Kasper alloys. ${ }^{10,11}$ The

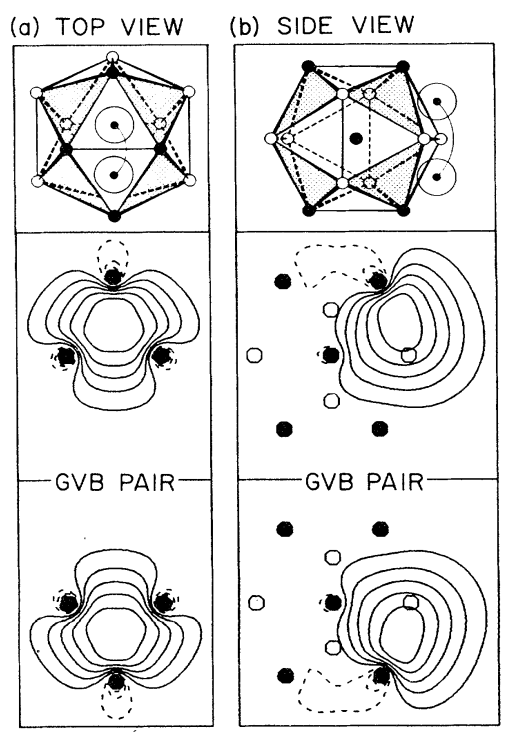

FIG. 4. GVB orbitals for one of the five primary resonance structures of icosahedral $\mathrm{Li}_{13}{ }^{+}$. The atoms are marked by circles; dots jointed by thin lines represent bondpaired electrons. Only one of the six equivalent bond pairs is shown (0.015-a.u. contour increments). Shaded triangular faces do not contain orbitals. 
present rules may require modification for metallic structures possessing severely distorted tetrahedra (e.g., body-centered cubic) and for structures containing insufficient tetrahedra to accommodate all valence $s p$ electrons (e.g., rule $\epsilon$ ).

The above principles are consistent with the relative energies and suggest that the optimum structures of small clusters should maximize the number of tetrahedra. However, for the icosahedron, the twenty tetrahedra all share the same central bulk atom, violating rule $\zeta$. This led us to predict several new $\mathrm{Li}_{13^{k+}}$ optimum-tetrahedral (OPTET) clusters that maximize the number of tetrahedra while satisfying rule $\zeta$. Each new structure ${ }^{6}$ has fifteen tetrahedra and is lower in energy than $I_{h}$. The best (Fig. 5) has a total energy $0.58 \mathrm{eV}$ lower than the icosahedron $(45 \mathrm{meV} / \mathrm{atom})$ ! These OPTET clusters generally lead to local fivefoldsymmetry axes, and since exact fivefold symmetry is inconsistent with three-dimensional crystal structure, OPTET bonding could play an important role in the stabilization of amorphous structures. ${ }^{6}$

Larger OPTET clusters are constructed by the addition of atoms to concave surface sites of the smaller OPTET clusters, forming at least two new tetrahedra for each atom added. The $\mathrm{Li}_{26}$ OPTET cluster is isostructural with the $\mathrm{Cu}_{10} \mathrm{Zn}_{16}$ cluster of $\mathrm{Cu}_{5} \mathrm{Zn}_{8}(\gamma-$ brass) ${ }^{9}$ (both contain 57 tetrahedra). The $\gamma$-brass structure is composed of these 26-atom clusters centered at body-centered-cubic (bcc) lattice positions. The $\gamma$-brass alloys usually have a valenceelectron/atom ratio of $21 / 13$, although an alloy of approximate composition $\mathrm{Ag}_{3} \mathrm{Li}_{10}$ also forms the $\gamma$-brass structure. ${ }^{10}$ Maximizing the number of tetrahedra also seems to be important for the stability of many other alloy structures. ${ }^{11}$

With such small clusters, surface effects are quite

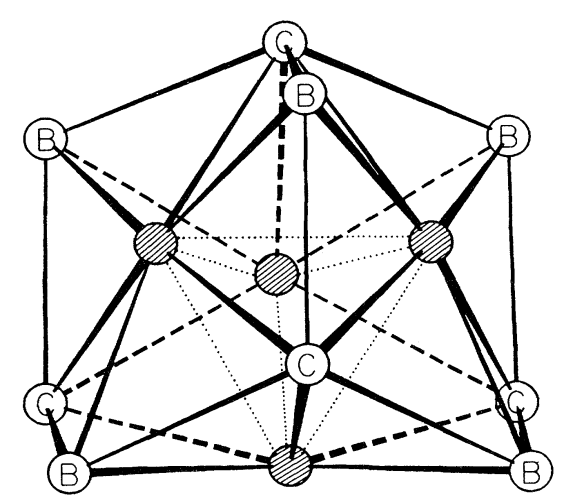

FIG. 5. The lowest-energy $\mathrm{Li}_{13}+$ OPTET isomer. This cluster consists of a central tetrahedron (shaded circles, dotted lines), four capping atoms (C), and five bridging atoms (B). This leads to a $C_{2}$-symmetry axis, two reflection planes, and five local $C_{5}$-symmetry axes. large so that planar clusters (which lead to an increased number of internal sites) are quite competitive. We have found a planar $\mathrm{Li}_{13}{ }^{+}$cluster that has a total energy $0.18 \mathrm{eV}$ lower than $I_{h}$ (but $0.40 \mathrm{eV}$ higher than the OPTET cluster).

These considerations suggest that there may be several regimes of structure for metal clusters. The smallest clusters either form planar structures (as in $\mathrm{Li}_{4}$ and $\mathrm{Li}_{6}$ ) or have very-low-energy planar configurations. For intermediate size, the OPTET structures are stable (e.g., $\mathrm{Li}_{8}, \mathrm{Li}_{13}{ }^{+}$, and perhaps extending well into the 20's), while very large clusters may yield structures approaching bulk character (hcp, fcc, bcc). The surface reactivities ${ }^{12}$ of these clusters can be expected to show dramatic dependences on the cluster regime (e.g., whether planar, OPTET, or high symmetry is favored).

Next, we apply these GVB ideas to close-packed (hcp and fcc) bulk metals and show how to rationalize certain solid solubilities and alloys in terms of electron/atom ratios.

Close-packed (cp) systems have twice as many tetrahedra as atoms. For the $\mathrm{Be}$ and $\mathrm{Zn}$ columns, the GVB model suggests that the optimum electronic structure be visualized in terms of one valence electron per tetrahedron. For a cp alkali or noble metal, only half the tetrahedra need be occupied by electrons, making the cp metals far more stable than the small cp clusters (where all tetrahedra are occupied, some with two electrons). Our rules do not state which cp structure is favored for these systems. Indeed, the alkali metals $^{13,14}$ and the alkaline-earth metals ${ }^{13}$ seem to have nearly identical energies for hcp, fcc, and bcc. The noble metals $(\mathrm{Cu}, \mathrm{Ag}$, and $\mathrm{Au})$ are all fcc while $\mathrm{Zn}$ and $\mathrm{Cd}$ are hop.

For cp systems with three valence electrons per atom, half the tetrahedra must be doubly occupied, while the other half are singly occupied. In fcc, each tetrahedron shares edges with six other tetrahedra, whereas pairs of tetrahedra are face shared in hcp. On the basis of rule $\delta$, we conclude that fcc should be strongly favored over hcp, and indeed, neither B, Al, $\mathrm{Ga}$, nor In leads to hcp, while Al leads to fcc and In to a slightly distorted fcc.

With four valence electrons per atom, all tetrahedra would be doubly occupied, strongly disfavoring either cp structure (rules $\gamma$ and $\delta$ ). Indeed, except for $\mathrm{Pb}$, the group-IV elements are either nonmetals (with strong two-electron covalent bonds) or metals with low coordination numbers. It is well known that $\mathrm{Hg}, \mathrm{Tl}$, $\mathrm{Pb}$, and $\mathrm{Bi}$ exhibit properties suggesting that the $(6 s)$ pairs are particularly stable and not easily hybridized with the $p$ orbitals to form normal $s p^{3}$ hybrids, perhaps rationalizing the fcc form for $\mathrm{Pb}$.

Rules $\gamma$ and $\delta$ place upper limits of $2 e^{-}$/atom for hcp and $3 e^{-/} /$atom for fcc. These limits are consistent 
with known alloy formations and solid solubilities, ${ }^{10,15}$ e.g., comparing atoms with similar sizes $\left[\mathrm{Ag}\left(1 e^{-}\right)\right.$, $\mathrm{Zn}\left(2 e^{-}\right), \mathrm{Al}\left(3 e^{-}\right)$, and $\mathrm{Sn}\left(4 e^{-}\right) ;$metallic $\operatorname{radii}^{13}$ $1.44,1.39,1.43$, and $1.54 \AA$, respectively], $\mathrm{Ag}$ and $\mathrm{Zn}$ are both highly soluble in fcc Al (23.8 and 66.5 at. \%, respectively), while the solubility of $\mathrm{Sn}$ in $\mathrm{Al}$ is only 0.02 at. \%. Similarly, the solubilities of $\mathrm{Al}$ and $\mathrm{Sn}$ in hcp $\mathrm{Zn}$ are small (2.4 and 0.14 at. \%, respectively), while $\mathrm{Ag}$ is 5 at. \% soluble in $\mathrm{Zn}$ and forms hcp-like alloys with up to $63.8 \% \mathrm{Ag}\left(\mathrm{Ag}_{5} \mathrm{Zn}_{8}\right.$ forms $\gamma$-brass).

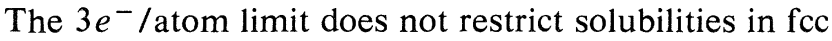
$\mathrm{Ag}\left(1 e^{-/ \text {atom }}\right)$; hence, the solubilities are all high ( $\mathrm{Zn}, 40.2$ at. \%; $\mathrm{Al}, 20.34$ at. \%; Sn, 11.5 at. \%).

We believe that these ideas of interstitially localized singly occupied orbitals will form a useful starting point in developing valence-bond ideas for predicting geometries, electronic structure, and properties of metallic clusters, and for describing localized states in metals and alloys such as defects or interfaces. These ideas may also provide useful concepts for predicting transport properties and the chemistry and catalytic properties of such systems.

This work was partially supported by the National Science Foundation through Grant No. DMR8215650 .

${ }^{1} \mathrm{~L}$. Pauling, The Nature of the Chemical Bond and the Structure of Molecules and Crystals (Cornell Univ. Press, Ithaca, N.Y., 1960), 3rd ed.

${ }^{2}$ W. A. Goddard, III, T. H. Dunning, Jr., W. J. Hunt, and P. J. Hay, Accts. Chem. Res. 6, 368 (1973).

${ }^{3}$ W. A. Goddard, III, and L. B. Harding, Ann. Rev. Phys. Chem. 29, 363 (1978).

${ }^{4}$ G. T. Surratt and W. A. Goddard, III, Solid State Com- mun. 22, 413 (1977), and Phys. Rev. B 18, 2831 (1978).

5J. J. Barton, W. A. Goddard, III, and T. C. McGill, J. Vac. Sci. Technol. 16, 1178 (1979); C. A. Swarts, T. C. McTill, and W. A. Goddard, III, Surf. Sci. 110, 400 (1981); A. Redondo and W. A. Goddard, III, J. Vac. Sci. Technol. 21, 344 (1982); A. Redondo, W. A. Goddard, III, and T. C. McGill, Surf. Sci. 132, 49 (1983).

${ }^{6} \mathrm{M}$. H. McAdon and W. A. Goddard, III, to be published, and to be published.

${ }^{7}$ H.-O. Beckmann, J. Koutecký, and V. Bonačić-Koutecký, J. Chem. Phys. 73, 5182 (1980).

${ }^{8}$ Our results for $\mathrm{Li}_{4}$ are consistent with a pseudo-JahnTeller description (Ref. 7). However, the pseudo-JahnTeller description does not predict the analogous distortion for the $\mathrm{Li}_{6}$ ring.

${ }^{9}$ A. Taylor, X-Ray Metallography (Wiley, New York, 1961).

10W. Hume-Rothery and G. V. Raynor, The Structure of Metals and Alloys (Institute of Metals, London, 1962), 4th ed.

${ }^{11}$ F. C. Frank and J. S. Kasper, Acta Crystallogr. 12, 483 (1959), and 11, 184 (1958).

${ }^{12}$ M. D. Morse, M. E. Geusic, J. R. Heath, and R. E. Smalley, J. Chem. Phys. 83, 2293 (1985); R. L. Whetten, D. M. Cox, D. J. Trevor, and A. Kaldor, Phys. Rev. Lett. 54, 1494 (1985); S. C. Richtsmeier, E. K. Parks, K. Liu, L. G. Pobo, and S. J. Riley, J. Chem. Phys. 82, 3659 (1985).

$13 \mathrm{~J}$. Donohue, The Structure of the Elements (Wiley, New York, 1974).

${ }^{14}$ R. W. Overhauser, Phys. Rev. Lett. 53, 64 (1984); K. Takemura and K. Syassen, Phys. Rev. B 28, 1193 (1983); M. S. Anderson and C. A. Swenson, Phys. Rev. B 31, 668 (1985).

${ }^{15}$ M. Hansen, Constitution of Binary Alloys (McGraw-Hill, New York, 1958); R. P. Elliot, Constitution of Binary Alloys, 1st Supplement (McGraw-Hill, New York, 1965); F. A. Shunk, Constitution of Binary Alloys, 2nd Supplement (McGraw-Hill, New York, 1969); W. G. Moffatt, Constitution of Binary Alloys, 3rd Supplement (McGraw-Hill, New York, 1983). 